\title{
Les digues des réservoirs supérieur et inférieur de la station de pompage de Revin
}

\author{
par M. Ponsy \\ Chef de Service Etudes Pompages \\ R.E.H. Nord Plobsheim
}

\author{
et M. Londez \\ Ingènieur à Mécasol, Paris.
}

L'aménagement de l'usine hydroélectrique d'accumulation journalière par pompage de Revin a été présenté l'année dernière par M. Ponsy.

Nous rappellerons simplement ici ses principales caractéristiques. Nous vous présentons aujourd'hui les problèmes d'hydrologie et d'écoulement dans les digues du bassin supérieur et du bassin inférieur.

\section{Présentation des ouvrages}

L'aménagement est implanté dans le massif des Ardennes composé d'un plateau au voisinage de la cote 400 entaillé par de profondes vallées encaissées.

L'aménagement, du point de vue des digues (fig. 1) se compose d'un bassin supérieur entièrement artificiel, du type collinaire aménagé sur le plateau des Marquisades. Le bassin inférieur est créé par une digue d'une quarantaine de mètres de hauteur qui barre le lit d'un petit affluent de la Meuse.

Les volumes utiles des deux bassins sont de $7000000 \mathrm{~m}^{3}$ et la dénivellation brute varie en fonction du marnage de $220 \mathrm{~m}$ à $245 \mathrm{~m}$.

Le massif rocheux, support des ouvrages, est composé de schistes et de quartzites du Cambrien supérieur, superficiellement altérés mais sains en profondeur.

Le plateau des Marquisades, dans lequel l'altération a parfois pénétré de façon relativement importante, comporte également des lambeaux de formation tertiaire sabloargileux.

La vallée dans laquelle est placé le bassin inférieur est, par contre, directement creusée dans les schistes avec un faible recouvrement d'éboulis et d'alluvions torrentielles.

Le bassin supérieur (fig. 2) a été réalisé en écrêtant le plateau, avec utilisation directe des produits de déblai pour la confection des digues. La longueur des digues périmétrales est de $4200 \mathrm{~m}$, leur hauteur moyenne de $15 \mathrm{~m}$ et le volume global des terrassements de l'ordre de $4000000 \mathrm{~m}^{3}$.

Les digues sud ont une hauteur inférieure à $15 \mathrm{~m}$, et les digues nord supérieure à $15 \mathrm{~m}$ (fig. 3).

$\mathrm{La}$ digue du bassin inférieur (fig. 4) est du type noyau central imperméable en éboulis limoneux encadré de recharges provenant du marinage des galeries de l'usine souterraine et des adductions.

\section{Problèmes posés. Paramètres}

Les problèmes à résoudre pour la conception de ces digues sont directement liés à deux séries de paramètres fondamentaux :

- les caractéristiques hydrologiques et géotechniques du site;

- le marnage très rapide quotidien.

Le bassin inférieur ne présente pas de caractéristiques particulières en ce qui concerne l'hydrologie. Il se situe dans une formation schisteuse assez peu perméable dans la masse dont il a suffi de maîtriser les zones d'altération superficielle.

Le site du bassin supérieur présente un certain nombre de caractéristiques spécifiques qui ont nécessité une analyse détaillée tant au point de vue de la mécanique des 

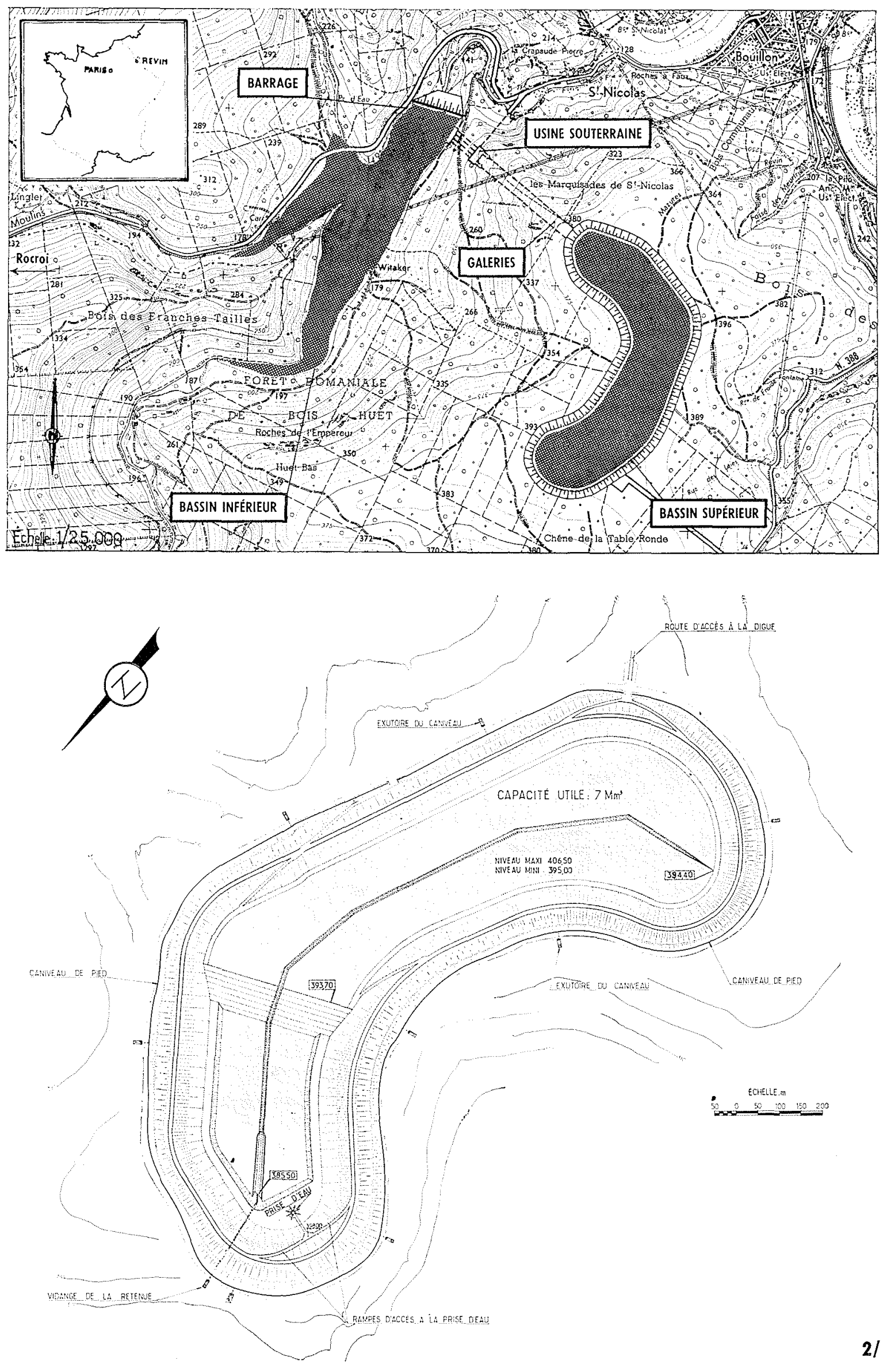


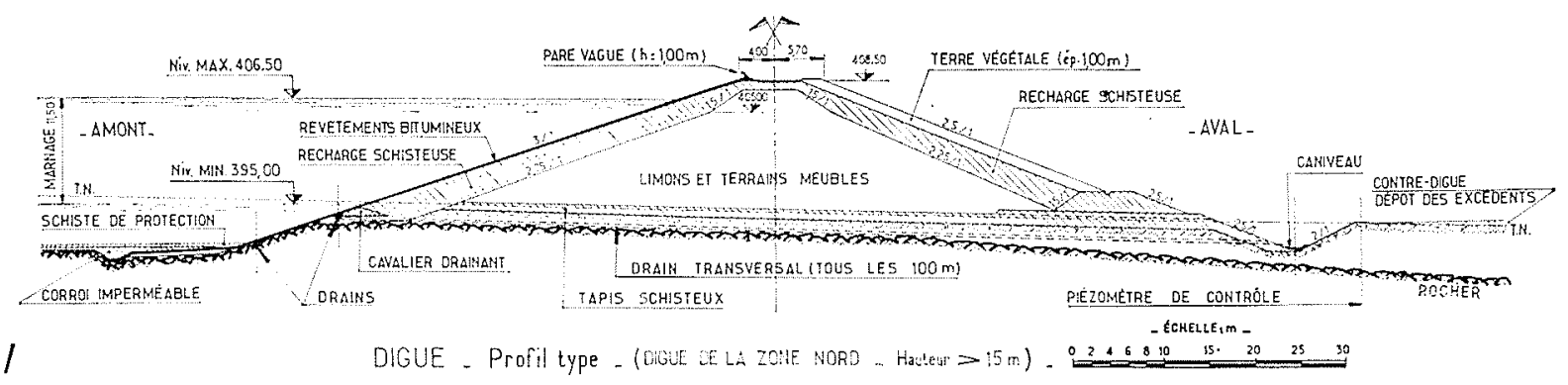

$3 a l$

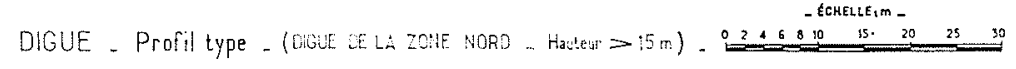

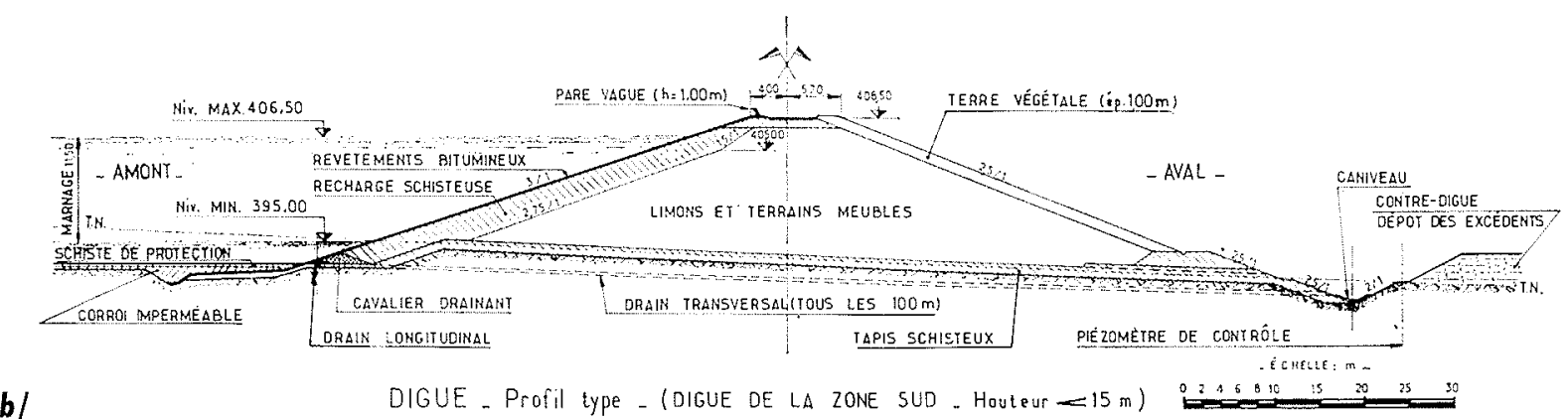

$3 b /$

DIGUE - Profil type - (DIGUE DE LA ZONE SUD . Houteur $-15 \mathrm{~m}$ )

1/ Plan d'ensemble de l'aménagement.

2/ Bassin supérieur.

Plan d'ensemble des digues.

3/ Bassin supérieur.

Coupes des digues.

\section{4/ Bassin inférieur.}

Plan d'ensemble et coupe type.

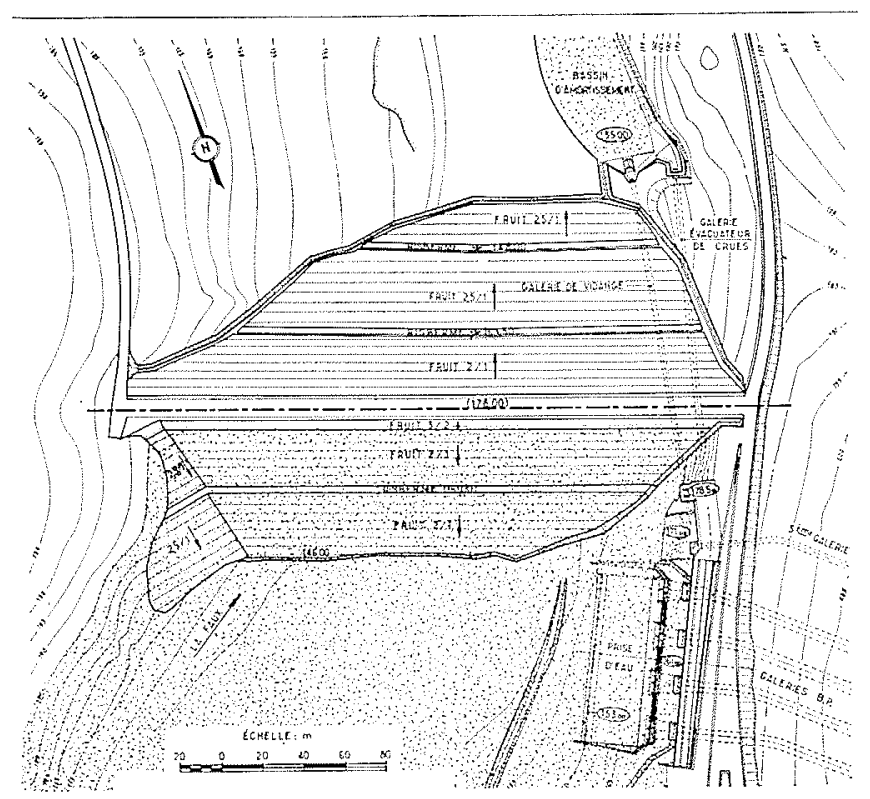

- AMONT

舟

- AVAL -
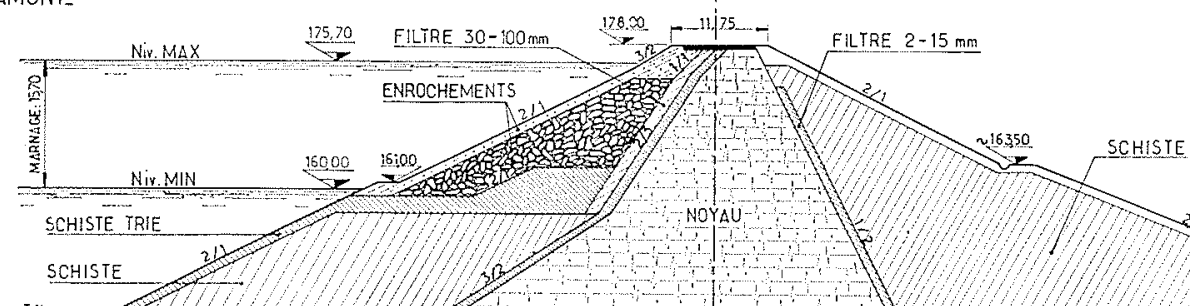

(1)

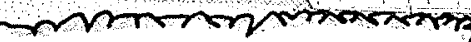


sols - assez grande dispersion et originalité des caractéristiques des matériaux - qu'au point de vue hydrologique, modification des conditions de nappes préexistantes. Nous reviendrons sur ces paramètres lors de l'analyse de chacun des ouvrages.

Nous abordons tout d'abord le caractère propre à l'aménagement: la vidange rapide quotidienne.

Les vitesses de vidanges sont très élevées: environ $2 \mathrm{~m} / \mathrm{h}$ dans les deux bassins. Ces valeurs sont beaucoup plus grandes que celles des vitesses de vidange habituelles dans les retenues normales de barrage.

On sait qu'un régime hydraulique étant établi dans un terrain, le passage à un régime différent - vidange rapide par exemple - par modification des conditions aux limites de l'écoulement, se fait à une vitesse, essentiellement liée à la perméabilité des matériaux constitutifs du sol.

Schématiquement, on peut définir diverses situations transitoires permettant de passer d'un régime d'écoulement à retenue pleine à celui, supposé permanent correspondant aux conditions aux limites de la vidange.

Une première phase correspond à une relaxation sans échange d'eau corrélative au déchargement du terrain par l'abaissement du plan d'eau: cette relaxation est rapide et produit une baisse des pressions interstitielles à l'intérieur de l'ouvrage. Dans une deuxième phase s'établit le nouveau régime d'écoulement, à une vitesse croissant avec la perméabilité du sol.

Si la diminution de pression dans la première phase ne permet pas d'assurer la stabilité du parement amont, ce qui est en général le cas, il faut augmenter la perméabilité pour accélérer la deuxième phase.

En conséquence, pour la réalisation des parements amont de digues soumises à des vidanges très rapides, le choix est limité aux conceptions extrêmes suivantes:

- recharge amont constituée de matériaux très perméables, tel que le niveau piézométrique y suive celui du plan d'eau;

- parement amont parfaitement imperméabilisé par un revêtement d'étanchéité de façon à soustraire le matériau sous-jacent à tout échange hydraulique.

La première solution a été retenue pour la digue du bassin inférieur qui comporte une importante recharge amont en enrochements, la deuxième pour les digues du bassin supérieur dont le parement amont est étanché par un masque de béton bitumeux.

Les choix ont été déterminés par des conditions essentiellement économiques.

\section{Méthodes de calcul}

La connaissance des régimes d'écoulement dans les digues est nécessaire pour les études de stabilité. Ces études sont faites sur la base de deux programmes de calcul : l'un que nous citons pour mémoire ici, étudie la stabilité par cercles de glissement. On y introduit toutes les informations hydrauliques nécessaires pour le calcul du coefficient de sécurité minimal.

L'autre, qui est utilisé en premier dans l'étude, est un programme de résolution numérique de l'équation de Laplace de régimes d'écoulement permanent dans les mi- lieux poreux par la méthode des différences finies. Ce programme résout numériquement aux nœuds d'un maillage calqué sur les éléments de l'étude de stabilité, l'équation de Laplace une fois définies les conditions aux limites du problème. Les potentiels étant calculés, le programme détermine en tout point les pressions $\mu$ et l'intégrale de ces pressions à la base et sur les faces latérales de tranches; ces résultats sont incorporés à l'étude de stabilité.

Ces calculs supposent les conditions aux limites figées. Parmi ces limites figurent les lignes de saturation. Celles-ci peuvent être déterminées à partir du même programme, par approximations successives, mais cette technique est trop lourde et onéreuse pour être utilisée systématiquement. Il est le plus souvent préférable d'utiliser des techniques plus simples à base d'analogie électrique sur papier conducteur ou sur réseau maillé, le papier conducteur s'avérant dans la plupart des cas la solution la plus satisfai sante, étant bien entendu qu'il ne s'agit pas pour nous de faire de la science mais d'avoir une approximation aussi satisfaisante que possible, dans des limites économiques raisonnables, des conditions aux bornes du problème; les erreurs possibles sur la position des lignes de saturation sont d'ailleurs relativement faibles et en tout cas peu importantes par rapport à l'ensemble des autres hypothèses qu'on est amené à faire. Je fais en particulier allusion à l'anisotropie des terrains mis en cuvre.

\section{Le bassin supérieur}

Le site du bassin supérieur comporte de bas en haut, en allant du résistant au meuble (fig. 5):

- le rocher-schistes et quarzites du primaire, géologiquement non altérés mais très feuilletés. II est peu perméable. A l'extraction, il ne permet pas de constituer des recharges suffisamment perméables pour résister aux conditions très sévères de marnage du bassin;

- le rocher superficiel - frange des schistes et quartzites géologiquement altéré sans avoir atteint le stade de l'argile - plus perméable en place que le rocher, car plus ou moins exfolié. Il est le siège d'une nappe. Il fournit à l'extraction un tout venant d'assez bonne qualité pour le corps aval des digues;

- les matériaux meubles constitués par des sables et argiles du tertiaire, de limons de surface et de matériaux fins provenant de l'altération des schistes.

Bien que ceci ne concerne pas directement le sujet traité ici, nous voudrions souligner les caractéristiques assez particulières de ce matériau superficiel d'origine schisteuse, il s'agit d'un produit d'altération des schistes se présentant sous la forme d'une farine ayant le comportement d'un silt avec un indice de plasticité variant de 0 à $5 \%$. Ce matériau est difficile à compacter en particulier du fait de son faible indice de plasticité comparé à sa granulométrie.

Pour la constitution des digues, aucun gisement d'enrochements, économiquement exploitable, n'existant à proximité du site, E.D.F. a été amenée à projeter une digue à masque amont étanche de béton bitumineux.

Les couches étanches surmontent une couche drainante très perméable d'enrobés bitumineux, elle-même plaquée sur une recharge rocheuse de qualité moyenne en tout venant 


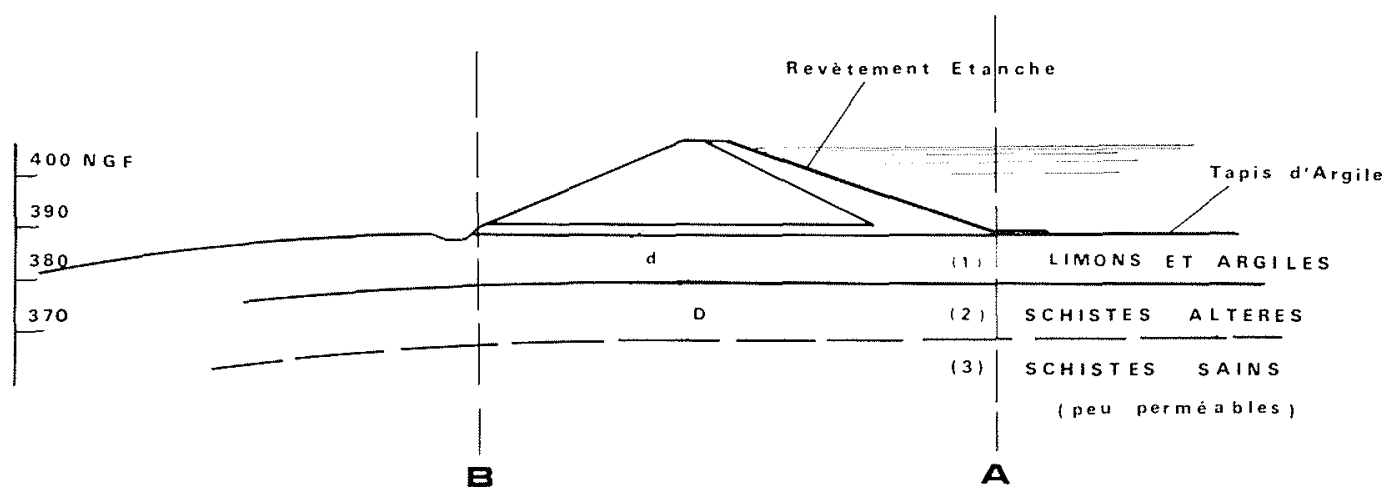

5/

Bassin supérieur.

Ecoulement sous digue.

Piézométrie dans $(2)$
\begin{tabular}{|c|c|c|c|}
\hline$d_{(m)}$ & $D_{(m)}$ & $H_{A}(m)$ & $H_{B}(m)$ \\
\hline 5 & 10 & 7.3 & 5.3 \\
\hline 10 & 10 & 6.3 & 5.0 \\
\hline
\end{tabular}

schisteux (fig. 3) extrait de la zone de surcreusement Nord, autour de la tulipe.

Le noyau des digues est constitué par les matériaux meubles soigneusement compactés; le parement aval est recouvert par un manteau de terre végétale anti-gel.

Les études de stabilité ont été conduites dans deux hypothèses :

- l'une dite fonctionnement normal, le masque amont remplissant parfaitement son rôle d'étanchéité;

- l'autre appelée fonctionnement accidentel qui suppose qu'une rupture du masque a provoqué la saturation de la recharge amont. Il est apparu nécessaire d'admettre que l'ouvrage devrait résister à une vidange rapide dans cette hypothèse. Comme on le verra plus loin, les débits de fuite peuvent alors rester limités, voire faibles, et de ce fait il est possible que l'alerte ne soit pas immédiate en dépit des dispositifs de surveillance prévus.

On a donc été conduit à étudier :

- d'une part, le régime des écoulements sous digues dans le cas normal;

- d'autre part, le régime des écoulements dans le complexe de la recharge amont dans le cas de la rupture du masque.

\section{Ecoulements sous digues.}

Le plateau à la cote 400 était, avant travaux, le siège d'une nappe locale uniquement alimentée par la pluie puisque le plateau est le point culminant de la région (fig. 5).

La nappe superficielle, essentiellement retenue par les formations peu perméables de surface, s'écoulait dans les schistes rocheux superficiels plus perméables et résurgeait à la périphérie du plateau.

La construction des ouvrages modifie les conditions aux limites de cet écoulement: le fond du bassin est imper- méabilisé par un corroi argileux $\left(\mathrm{K} \sim 10^{-8} \mathrm{~m} / \mathrm{s}\right)$ et à la mise en eau, le potentiel d'alimentation sera porté au voisinage de la cote 406,5 au lieu de 400 .

Le schéma de la figure 5 définit les conditions de calcul de ce régime hydraulique. Il faut noter la présence d'un tapis de schiste non altéré, tout venant compacté, dont l'une des fonctions est d'isoler le corps de digue de la piézométrie du terrain en place. Différentes hypothèses ont été envisagées concernant les épaisseurs relatives des divers horizons de la fondation; on n'a pas tenu compte, par prudence, de la possibilité de contrôler la piézométrie aval par des puits de décharge.

On peut noter qu'en pied amont la piézométrie des schistes altérés est toujours inférieure au poids de terre.

Cette étude a permis de définir les conditions de piézométrie en pied amont et aval des digues, pour la zone sud où l'épaisseur peu perméable est grande et pour les digues plus hautes de la zone nord dont le niveau de fondation est relativement proche de l'horizon plus perméable.

\section{Régime hydraulique dans la recharge amont.}

La structure du parement amont des digues et son dispositif de drainage sont définis par la figure 6.

Le noyau pratiquement imperméable de la digue est surmonté par une recharge en tout venant schisteux - de $2 \mathrm{~m}$ d'épaisseur - dont la perméabilité moyenne est de l'ordre de $10^{-4} \mathrm{~m} / \mathrm{s}$. Cette recharge constitue le support du revêtement bitumineux qui comporte:

- une couche filtrante de $4 \mathrm{~cm}$ d'épaisseur d'une perméabilité de $10^{-5} \mathrm{~m} / \mathrm{s}$;

- une couche drainante de $8 \mathrm{~cm}$ d'épaisseur de très forte perméabilité $\left(10^{-2} \mathrm{~m} / \mathrm{s}\right)$ (le binder);

- une bicouche étanche de $9 \mathrm{~cm}$ d'épaisseur totale, présentant une perméabilité inférieure à $5 \cdot 10^{-10} \mathrm{~m} / \mathrm{s}$. 


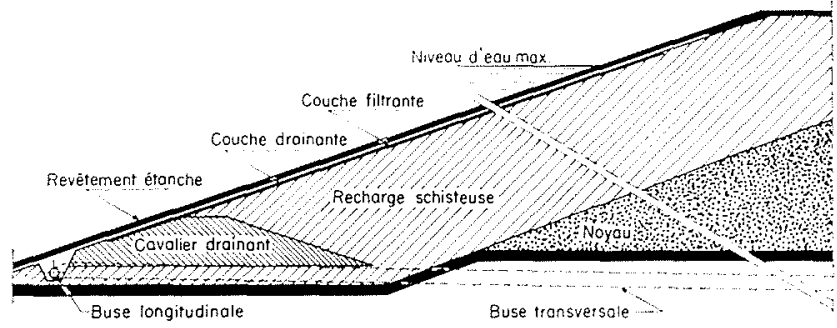

6/ Schéma de principe du parement pour l'étude des régimes d'écoulement dans la recharge amont.

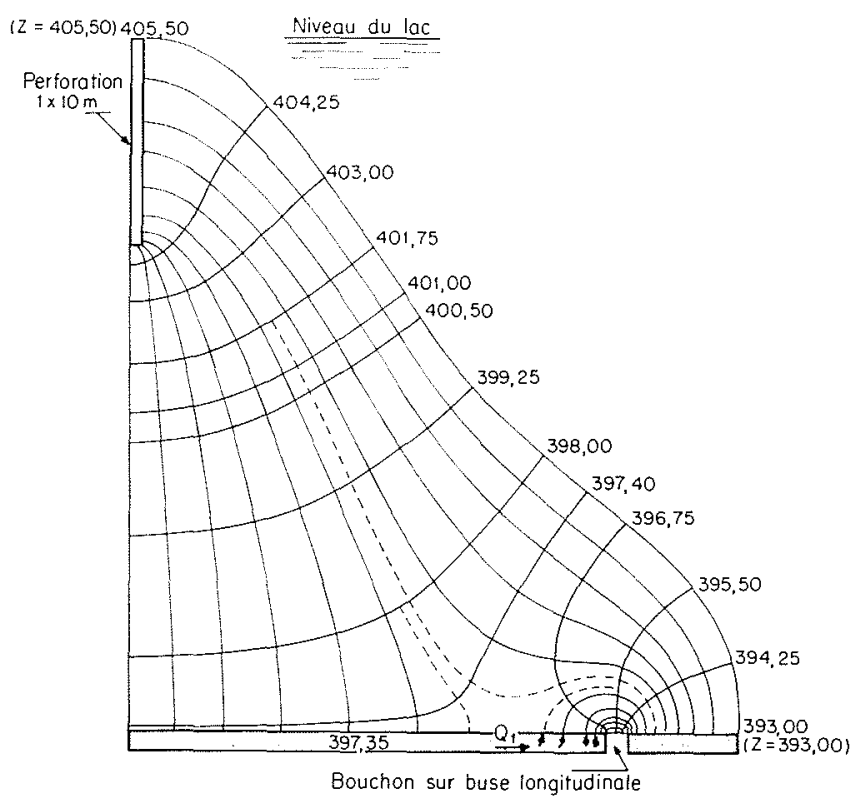

7/ Ecoulement dans un plan parallèle au talus.

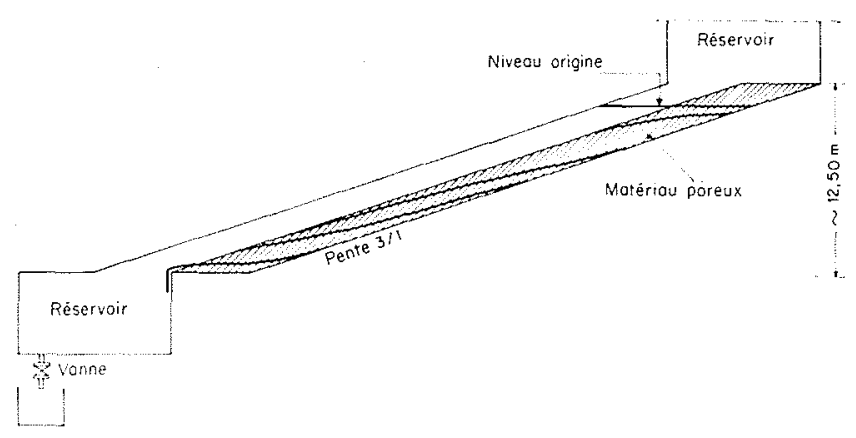

8/ Recharge schisteuse amont. Evolution de la surface libre.
La couche drainante enrobe à son pied un collecteur longitudinal de $0,20 \mathrm{~m}$ de diamètre qui débouche tous les $100 \mathrm{~m}$ dans des drains de $0,30 \mathrm{~m}$ de diamètre qui traversent la digue et débitent à l'aval dans le caniveau de drainage qui ceinture les digues. Ce caniveau comporte huit exutoires équipés de limnigraphes qui permettent de contrôler les fuites. Le réseau de buses de drainage est, par sécurité, systématiquement doublé par un drain de matériau très per. méable.

Le comportement de ce système hydraulique, qui n'est appelé à fonctionner que dans le cas d'un défaut d'étanchéité du revêtement a fait l'objet d'une série d'études qui ont été réalisées en collaboration avec le Cabinet d'études Ruby.

\section{Etude par aNALOGIE ÉLECTRIQUe.}

Elle avait pour but de définir les conséquences de l'ouverture d'une brèche dans le masque d'étanchéité et plus particulièrement de préciser dans quelle mesure la couche drainante et la recharge schisteuse pouvaient être saturées d'eau. Cette étude a été réalisée dans différentes hypothèses portant sur la dimension et la position de la perforation, l'écartement des drains transversaux et leur colmatage éventuel.

a) Le modèle à deux dimensions a permis de définir, dans un plan parallèle à la surface du parement, l'allure de l'écoulement en régime permanent tant dans le schiste que dans la couche drainante. La figure 7 donne la représentation d'un de ces réseaux d'écoulements.

On constate que les zones "mouillées" peuvent être très importantes mais que les débits restent faibles, sauf dans le cas d'une déchirure du revêtement en pied de talus. L'absence de gros débit dans le caniveau de pied n'est donc pas un indice sûr du bon état du revêtement.

b) La recharge schisteuse peut donc se trouver entièrement saturée d'eau et il importe de connaître les conditions d'essorage de cette recharge pendant la vidange rapide du bassin. Ce problème a été abordé également par analogie électrique; le modèle à deux dimensions, établi dans un plan transversal, permet d'obtenir dans ce plan la représentation d'un écoulement permanent. L'essorage a été considéré comme une succession de régimes permanents voisins correspondant à des niveaux de saturation décroissant dans le temps.

Cet essai a conduit aux résultats suivants :

- La couche drainante se vide très vite et reçoit du schiste un très faible débit; le soufflage des couches étanches n'est donc pas à craindre;

- Le débit d'essorage de la recharge schisteuse est pratiquement constant à partir du moment où la différence des niveaux de l'eau dans le schiste et la retenue atteint $1,50 \mathrm{~m}$ environ;

- Ce débit est faible et l'essorage est très lent, une douzaine d'heures environ alors que le temps de vidange du bassin n'est que de $6 \mathrm{~h}$. Ce résultat était prévisible, car la perméabilité de la recharge devrait être du même ordre que la vitesse de vidange du bassin pour que le niveau de saturation suive la baisse du plan d'eau de la retenue; ce qui n'est pas le cas ici où la perméabilité est de $10^{-4} \mathrm{~m} / \mathrm{s}$ pour une vitesse de vidange de $2 \mathrm{~m} / \mathrm{h}$. 
En conséquence, pour les calculs de stabilité du parenent amont, dans le cas "vidange rapide + défaillance de l'étanchéité du masque», il convient de retenir l'hypothèse que la recharge schisteuse est saturée.

\section{EtUde Par analogie VISQUeUSE.}

Les essais précédents présentent l'inconvénient de ne pas reproduire d'une manière continue le régime transitoire. Pour obtenir une visualisation de l'évolution de la surface libre de l'eau dans la recharge amont, l'étude de l'essorage dans un plan transversal de la digue a été reprise par similitude en analogie visqueuse. Le modèle est constitué par un canal vitré incliné dans lequel une couche de matériaux poreux représente la recharge, la partie libre correspondant à la retenue. L'appareil préalablement rempli d'huile est vidangé et on observe en fonction du temps la descente de la surface libre dans le matériau poreux. L'analogie de Hele Shaw permet de déduire ce qui se passe dans la recharge amont des digues. Les essais ont été réalisés pour plusieurs vitesses de vidange et pour différents niveaux initiaux de la retenue.

La figure 8 montre l'évolution de la surface libre dans le temps.

Ces expériences confirment bien les conclusions tirées des essais par analogie électrique, en particulier la très faible vitesse d'essorage de la recharge schisteuse.

Enfin, une dernière expérience a permis de simuler la formation de la zone de saturation à retenue pleine après perforation du revêtement, puis sa disparition pendant la vidange du bassin. L'écoulement dans un plan parallèle au parement amont est reproduit par analogie visqueuse entre plaques. L'évolution dans le temps des zones de saturation est représentée sur la figure 9.

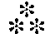

Ces diverses études ont conduit à une série de réseaux d'écoulement tant en fonctionnement normal qu'en fonc- tionnement accidentel dont nous vous présentons quelques exemples.

Cette hypothèse a été faite pour toutes les études de stabilité tant à l'aval qu'à l'amont. On a, en outre, conservé une sécurité : les puits de décharge non pris en compte dans les calculs.

Un réseau de piézomètres implantés dans et à l'extérieur de la digue permet de surveiller les niveaux piézométriques et de confronter les hypothèses retenues pour le dimensionnement des ouvrages avec l'évolution réelle du régime hydraulique. Des puits de décharge pourront alors éventuellement être réalisés pour améliorer le contrôle de la piézométrie.

\section{La digue du bassin inférieur}

La digue comporte (fig. 4):

a) Un noyau central en terre imperméable composé de limons graveleux à granulométrie continue, présentant des caractéristiques d'étanchéité satisfaisantes. Ces matériaux sont extraits d'un éboulis de pente proche du site et sont bien compactables.

b) Une recharge rocheuse aval constituée par le mareing provenant de différentes excavations de l'Aménagement.

c) Une recharge rocheuse amont constituée:

- par des enrochements de carrière dans la zone du marnage rapide, c'est-à-dire sur le tiers supérieur. Leur perméabilité est suffisante pour que le niveau piézométrique à l'intérieur du matériau suive à tout instant le niveau de la retenue;

- par des schistes de marinage dans la partie basse de l'ouvrage. Ces matériaux ont des caractéristiques très moyennes mais suffisantes dans cette zone qui n'est

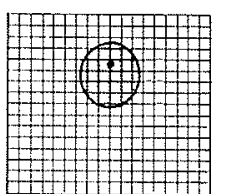

$T=7$

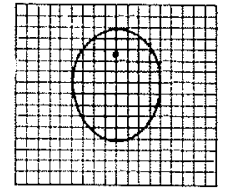

$T=19^{\prime \prime}$

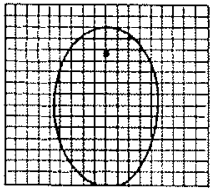

$T=32^{11}$

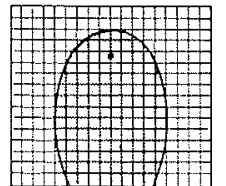

$T=41^{\prime \prime}$

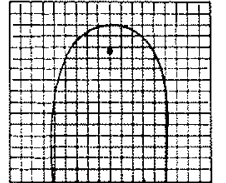

$\mathrm{T}=59^{\prime \prime}$

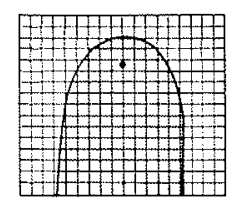

$T=2^{\prime} 11^{\prime \prime}$
Formation de la zone de saturation

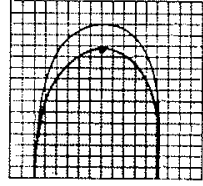

$T^{\prime}=7^{\prime \prime}$

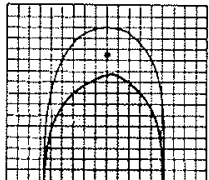

$T^{\prime}=13^{\prime \prime} 5$
Analogie visqueuse dans le plan parallèle au talus dans le binder.
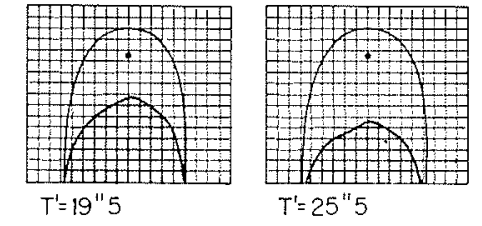

$T^{\prime}=25^{t \prime} 5$

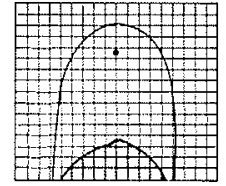

$T^{\prime}=31^{\prime \prime}$

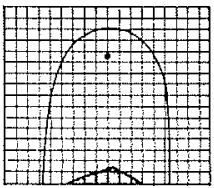

$T^{\prime}=37^{\prime \prime}$
Essoroge

Tcorreat $=5 \mathrm{~cm}$ sur le modèle

Echelle du modele $1 / 15^{2}$ 


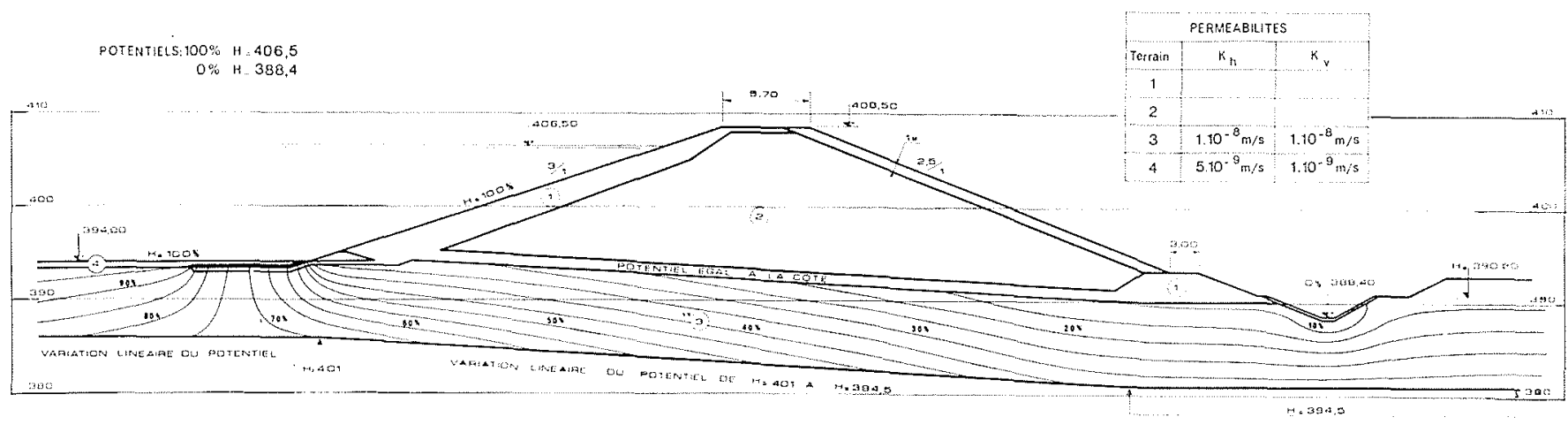

10/ Aménagement đe Revin. Bassin supérieur. Réseau d'écoulement. Digue Moyenne. Fonctionnement normal. Retenue pleine.

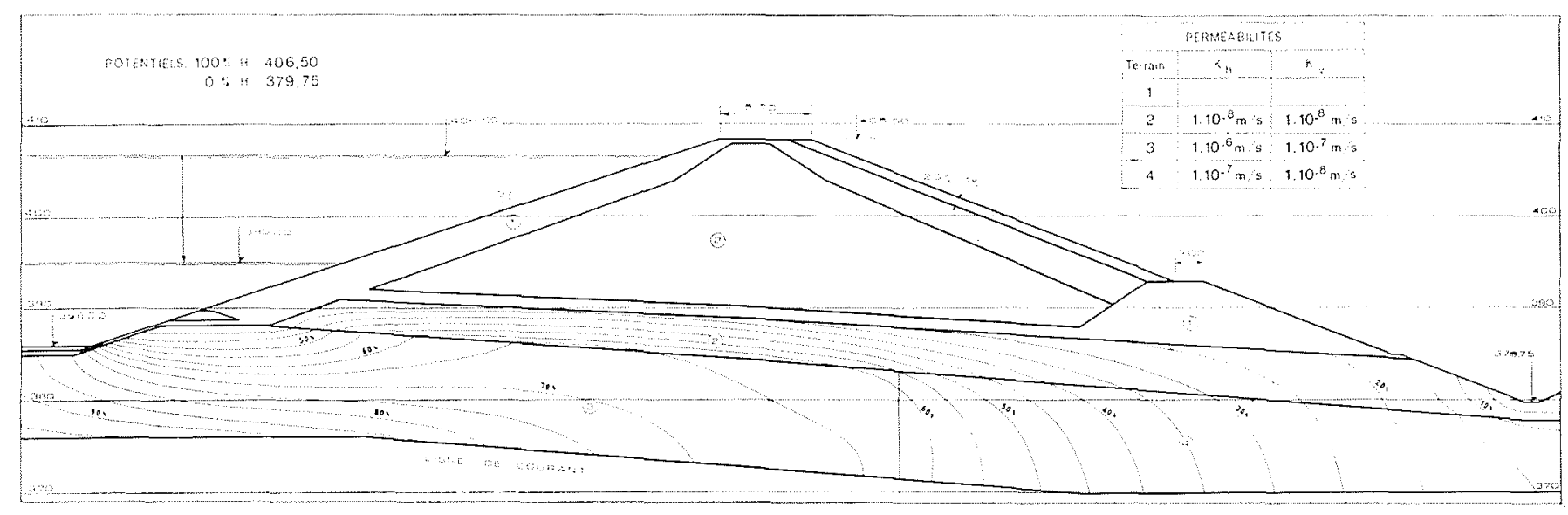

11/ Aménagement de Revin. Bassin supérieur. Réseau d'écoulement. Digue haute. Fonctionnement normal. Régime permanent.

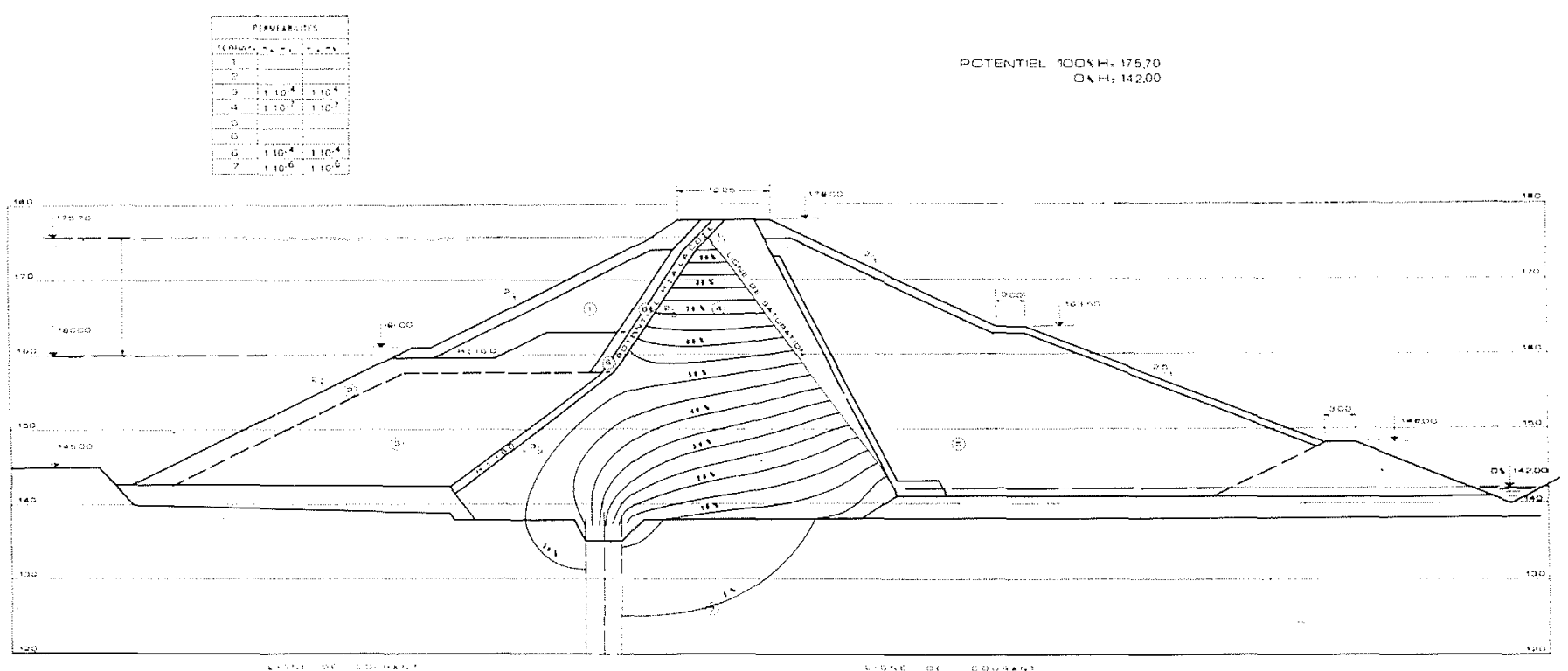

12/ E.D.F. REH Nord. Aménagement de Revin. Bassin inférieur. Exemple du réseau d'écoulement. Vidange rapide classique à 160 NGF. 
sollicitée que par les vidanges exceptionnelles qui s'effectuent lentement.

Le choix de cette solution avait l'avantage de limiter les travaux à un chantier de terrassement; par rapport à la solution masque amont, elle était compétitive en dépit du coût élevé des enrochements importés de carrières éloignées, le volume de ces enrochements étant relativement faible.

- Du point de vue hydrologique, il s'agit de connaître l'évolution de la pression interstitielle dans le noyau pendant les vidanges.

Nous avons fait l'hypothèse que la piézométrie à l'intérieur du noyau n'est pas modifiée pendant la durée de la vidange, soit environ huit heures.

En d'autres termes, les calculs de stabilité sont faits en prenant comme pression interstitielle dans le noyau les pressions correspondant à la retenue pleine. Il est évident que cette condition n'est mathématiquement pas rigoureuse puisque la pellicule du noyau au contact des filtres se trouve très rapidement à une piézométrie nulle; mais on peut démontrer que la propagation de la dissipation de la pression interstitielle à l'intérieur du noyau est lente au regard de la rapidité des marnages: on peut, en effet, l'estimer à environ $30 \mathrm{~cm}$ par jour.

- L'hypothèse faite revient à admettre la non variation de la résistance au cisaillement pendant la vidange. En effet, les calculs de stabilité effectués admettent que la contrainte totale $\sigma$ ne dépend que de la charge à la verticale du point étudié. Or, le contact noyau-recharge dans le cas particulier étudié, est dans une zone où les variations du plan d'eau ne modifient pratiquement pas la charge verticale. La pression interstitielle u étant supposée constante, la contrainte intergranulaire $\sigma^{\prime}=\sigma-u$ reste donc constante.

Les calculs nous ont montré la nécessité de casser le parement amont du noyau pour augmenter l'épaisseur de recharge perméable.

- Du point de vue technique de calcul, nous avons déterminé les pressions dans le noyau en régime permanent et transposé ces valeurs dans le programme de vidange rapide pour obtenir l'état qui nous intéressait.

L'hypothèse faite n'est pas rigoureuse car la variation de la charge d'eau influence tout le corps de digue; la contrainte totale diminue donc pendant la vidange d'une valeur d'ailleurs faible compte tenu de la configuration de la digue. En tout état de cause, la variation d'indice des vides du noyau reste faible pendant la vidange et la résis. tance au cisaillement ne varie pratiquement pas.

Pour conclure et à titre d'exemple la figure 12 représente le réseau de vidange rapide classique qui s'établirait si le bassin inférieur était laissé à la cote 160 pendant quelques jours (c'est-à-dire bassin supérieur plein).

\section{Discussion}

présidents: MM. J.-C. Van Dam et J. Florentin

M. le Président VAN DAM remercie les deux conférenciers pour leurs intéressants exposés et ouvre la discussion:

M. Post fait la remarque suivante :

M. PONSY a montré tout l'intérêt de tuyaux en plastique (PVC) pour augmenter la capacité de drainage sous la digue et sous le revêtement en béton bitumineux. Si je suis convaincu que ces buses en plastique sont très utiles dan: la conception du drainage des petits barrages, comme l'a indiqué $M$. Dunglas ce matin, je pense toutefois qu'il faut rester très prudent quant à l'emploi dans des digues de grande hauteur en raison des déformations diverses qui peuvent les rompre. Cela s'est produit notamment pour les buses enterrées collectant le débit des puits filtrants du barrage de Grenada (U.S.A.), buses qu'il a fallu injecter et remplacer par un caniveau à ciel ouvert.

En réponse, $M$. Ponsy indique que la raison essentielle qui a fait choisir un plastique comme matériau constitutif des drains réside dans l'acidité des terrains en place; leur PH est de 2,5 à 3. D'autre part, on a tenu à avoir la continuité du fil d'eau dans les drains, ce qui est facile à réaliser si ceux-ci sont en matière plastique. Nous avons fait des essais de résistance jusqu'à des pressions de $30 \mathrm{~m}$ d'eau et les tuyaux se sont très bien comportés.

Il se posait, de plus, le problème de la résistance à la température de ces tuyaux lorsqu'ils devaient être enrobés dans le béton bitumineux (drain de pied du ievêtement). En effet, au-dessus de $80^{\circ} \mathrm{C}$ le plastique se déforme et perd ses caractéristiques mécaniques; le problème a été résolu en enveloppant les tuyaux dans une feuille de «Bidim» qui assure une isolation thermique suffisante pour permettre l'enrobage à chand des tuyaux.
Certaines buses en matière plastique, utilisées non pas comme drains mais comme conduites d'amenées d'eau, observe M. RUEFF, ont donné lieu à quelques difficultés par suite de leur déformation sous l'action successive de leur échauffement par le rayonnement solaire et de leur refroidissement par l'eau qu'elles transportent.

M. JEanpierre (E.D.F., R.E.H. Alpes-Nord) présente deux observations :

- La première pour illustrer quelques propos de M. Post énoncés au cours de la matinée: au bassin supérieur de Revin, on a effectivement dissocié la fonction collecte des eaux par les drains constitués de matériaux gradués, de la fonction évacuation de ces eaux qui est confiée à des conduites. Cette distinction apparaît particulièrement nécessaire aux constructeurs de canaux où les surfaces à évacuer sont grandes et où souvent les points de rejets sont éloignés les uns des autres.

- La seconde pour signaler que les recharges aval des digues supérieures de Revin comportent par endroit un drainage de celles-ci, réalisé par de petits drains de plastique de $3 \mathrm{~cm}$ de diamètre, disposés à divers niveau du remblai dans le sens amont-aval et destinés à éviter les effets d'une anisotropie probable du matériau à cet endroit.

M. le Président Van Dam remercie vivement les deux conférenciers et les personnes qui sont intervenues dans la discussion. Il donne ensuite la parole au Docteur B. Gilc pour l'exposé de sa communication. 